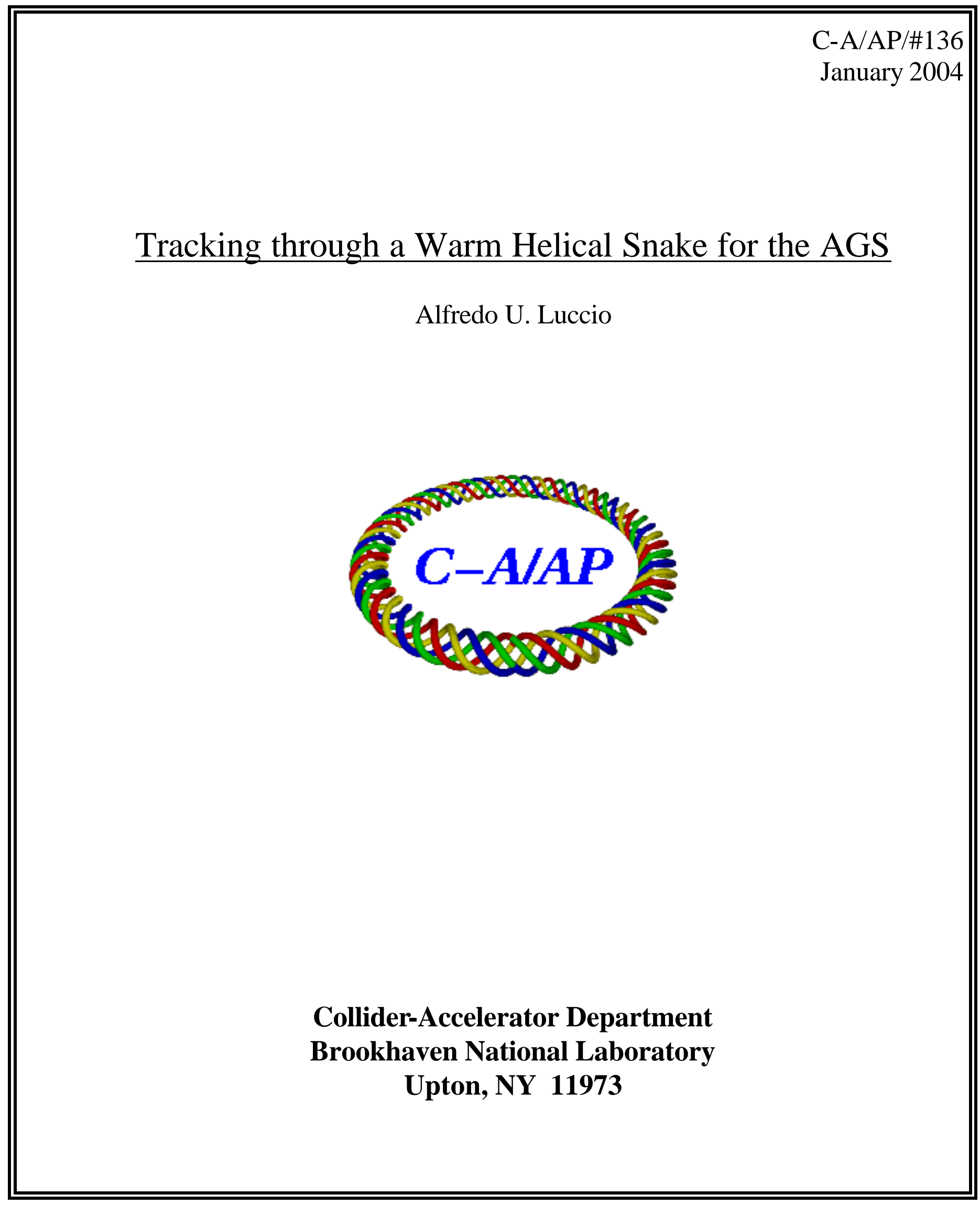




\title{
Tracking through a Warm Helical Snake for the AGS
}

\author{
Alfredo U. Luccio
}

January 30, 2004

\section{The Warm Snake}

A normal conducting helical warm snake has been designed and built in Japan [1] for the AGS. This is a $3 \mathrm{~m}$ long, 1.5 Tesla twisted dipole, with helical windings bent in a way to produce three sections with two different pitches. The design is similar to the AGS superconducting "Cold" snake [2], [3]. The optimization procedure of the field design in order to obtain vanishing small field integrals, was based on the same fitting model as for the Cold Snake described in a previous technical report [4].

We tracked with the program $S N I G$ [5] particle trajectories through the numerical map calculated for the snake with the code OPERA [6] and furnished to us by the snake designers. SNIG calculates also the spin evolution by integration of the BMT equation. The orbit transverse $4 \times 4$ matrix and $3 \times 3$ spin transfer matrix through the snake are calculated, for different proton energies, from the trajectories of some particles, as a Jacobian of the transformation. The orbit matrices will be used to match the snake into the AGS lattice, using the machine optics code $M A D$ [7]. The spin rotation angle and the direction of the spin rotation axis can be calculated from the spin matrix [8].

The field and field integrals on axis after optimization are shown in Fig. 1. The helical pitch from the map, i.e. $\tan \left(B_{a, y} / B_{a, x}\right)$, where $\overrightarrow{B_{a}}$ is the magnetic field on axis, is shown in Fig. 2.

The helical snake is focusing in both transverse planes $x$ and $y$. The focusing can be characterized by the two orbit matrix elements $T_{2,1}$ and $T_{4,3}$. To characterize the coupling we add up the square of the appropriate orbit matrix elements, forming the quantities

$$
\left\{\begin{array}{c}
U R=T_{1,3}^{2}+T_{1,4}^{2}+T_{2,3}^{2}+T_{2,4}^{2} \\
L L=T_{3,1}^{2}+T_{3,2}^{2}+T_{4,1}^{2}+T_{4,2}^{2}
\end{array} .\right.
$$

\section{Constant coil current. Nominal field of $1.5 \mathrm{~T}$.}

With $S N I G$ we run a series of cases at constant coil current, or at constant "nominal" field, for different beam energies. The spin rotation angle is maximum at the lowest energy: $\mu=15.06 \mathrm{deg}$, corresponding to a $8.4 \%$ snake, as shown in Fig. 3. Table 1 shows $\mu$ and the direction angles of the spin rotation axis, $\theta$, azimuth, with respect to $\vec{x}$ and $\phi$, elevation. The corresponding orbit parameters are shown in Table 2. The snake is focusing in both planes. It also generates coupling between the transverse planes. Coupling is in good part due to the longitudinal component of the magnetic field along the orbit, largest at the lowest energy. Focusing and coupling parameters, defined by Eq.(1), decrease with increasing energy, as shown in Fig. 4.

Trajectories at different energies are shown in Fig. 5. Spin component evolution at different energies is shown in Fig. 6. The field on the trajectory for two extreme energies is in Fig. 7. Orbit matrices for different energies are given in Table 6 . 


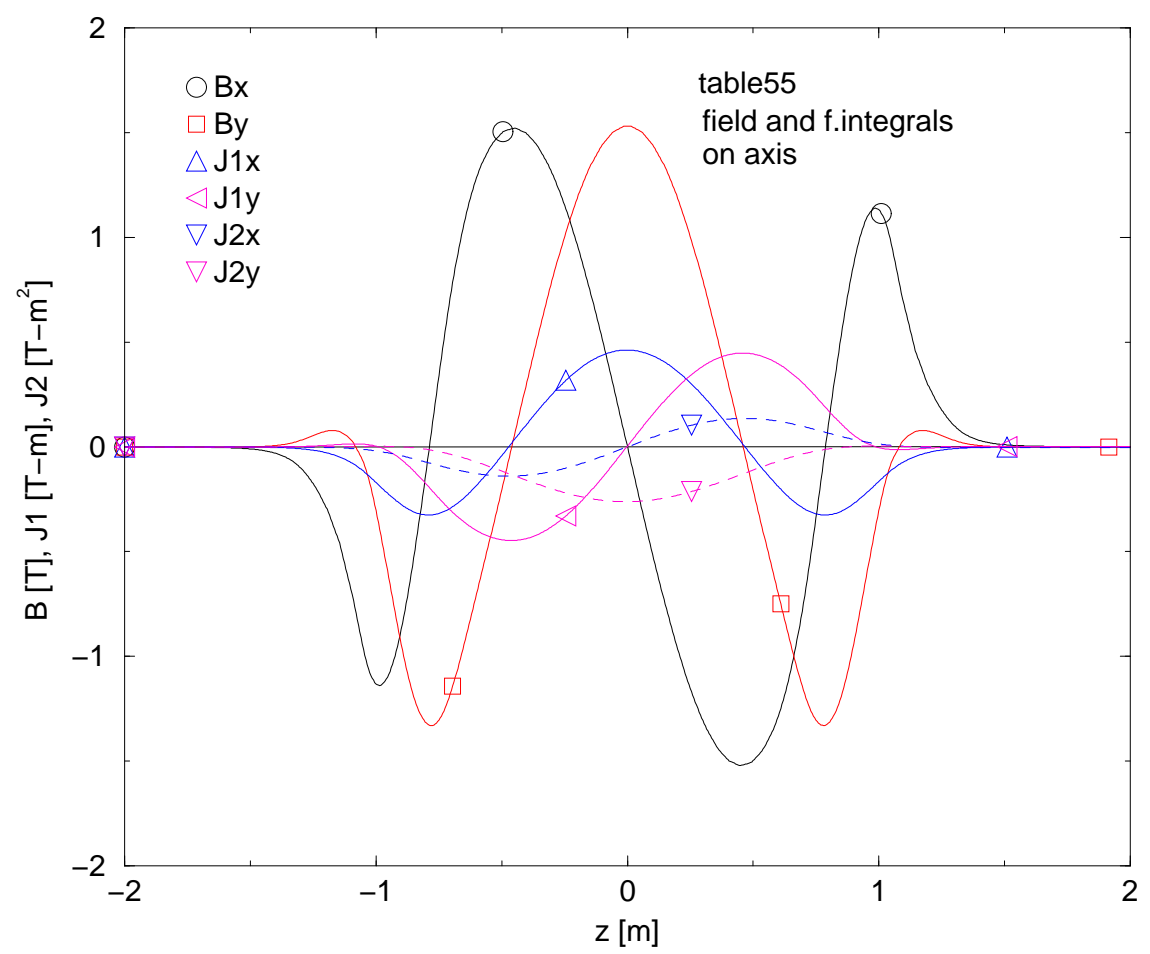

AUL 030519-001

Figure 1: Field on axis and field integrals for the final optimized geometry.

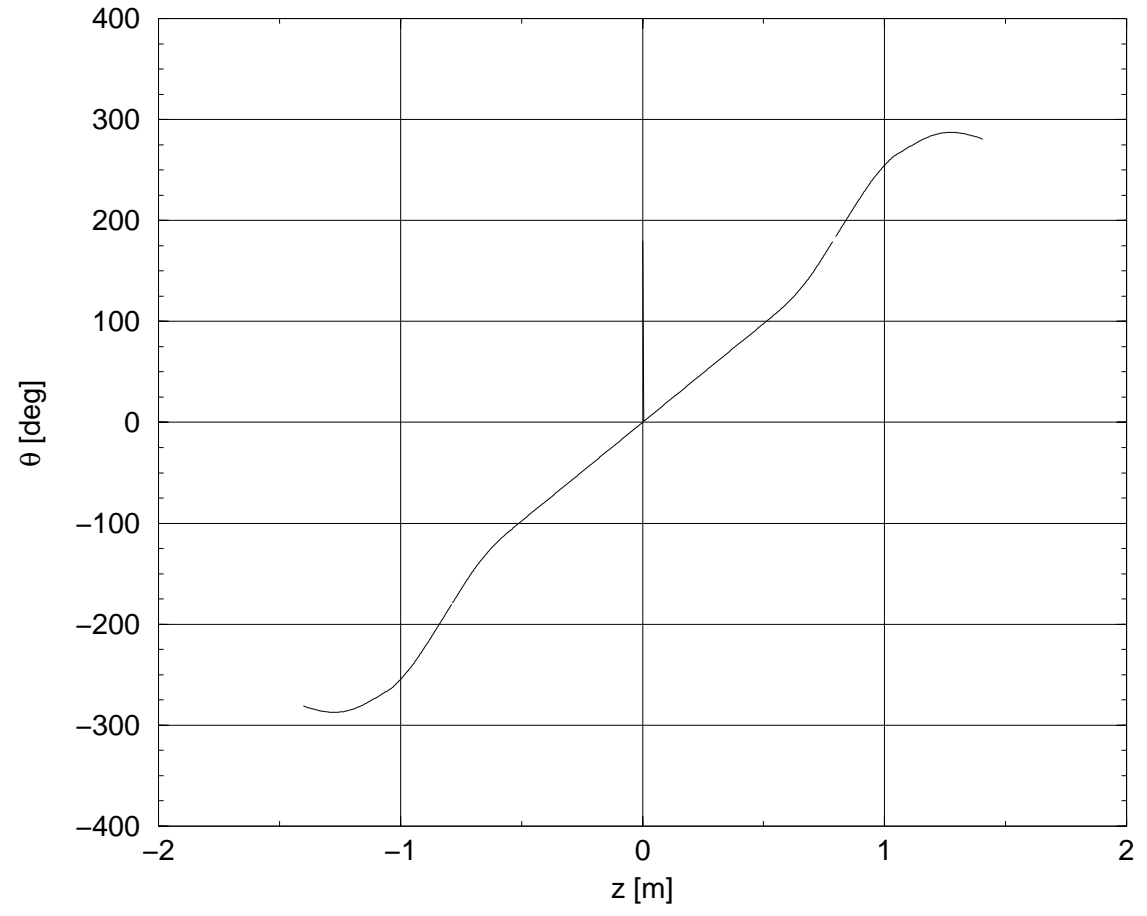

AUL 040129-02

Figure 2: Pitch field angle on axis showing two different pitches in three sections of the snake. 


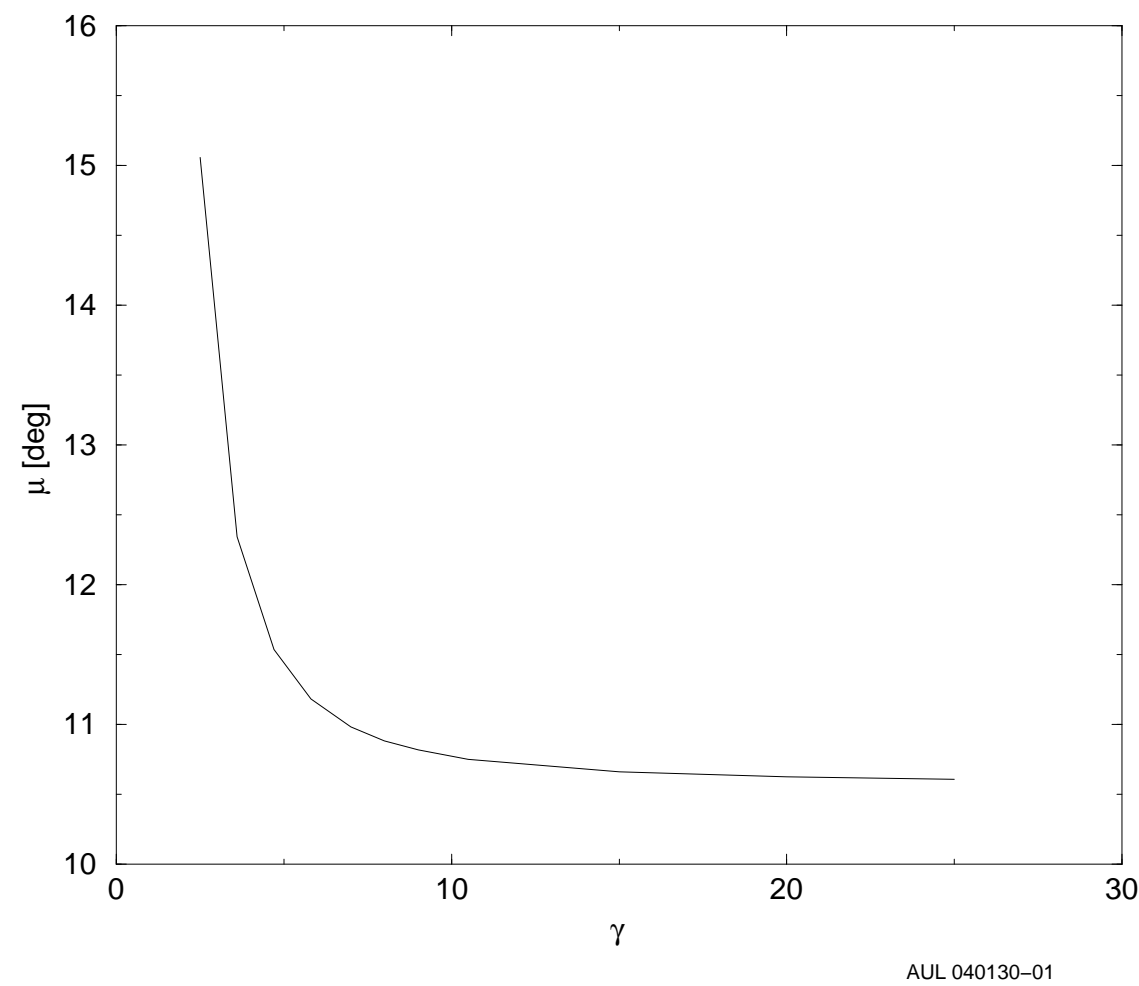

Figure 3: Spin rotation angle $\mu$ vs. beam energy. Constant coil current. Nominal field

Table 1: Spin rotation angle and axis angles vs. energy. Constant coil current. Nominal field

\begin{tabular}{rrrr}
$\gamma$ & $\mu[\mathrm{deg}]$ & \multicolumn{2}{c}{ axis $\theta, \phi[\mathrm{deg}]$} \\
2.5 & 15.0602924 & 180. & 0.0000515 \\
3.6 & 12.3459592 & 180. & 0.0000471 \\
4.7 & 11.5378547 & 180. & 0.0000463 \\
5.8 & 11.1840562 & 180. & 0.0000452 \\
7.0 & 10.9839858 & 180. & 0.0000445 \\
8.0 & 10.8847653 & 180. & 0.0000439 \\
9.0 & 10.8181221 & 180. & 0.0000437 \\
10.5 & 10.7526966 & 180. & 0.0000437 \\
15.0 & 10.6622784 & 180. & 0.0000434 \\
20.0 & 10.6250013 & 180. & 0.0000434 \\
25.0 & 10.6078946 & 180. & 0.0000433 \\
\hline
\end{tabular}

Table 2: Orbit lengthening and max excursion vs, energy. Constant coil current. Nominal field

\begin{tabular}{rrrr}
\hline$\gamma$ & $d s[\mathrm{~mm}]$ & \multicolumn{2}{c}{$\max x$ and $y[\mathrm{~mm}]$} \\
2.5 & 3.4638626 & 18.7709455 & 19.4545838 \\
3.6 & 1.5114265 & 12.4579206 & 12.8468039 \\
4.7 & 0.8553835 & 9.3668346 & 9.6699317 \\
5.8 & 0.5521877 & 7.5132382 & 7.7709462 \\
7.0 & 0.3752853 & 6.2050619 & 6.4070917 \\
8.0 & 0.2858546 & 5.3525803 & 5.5920703 \\
9.0 & 0.2250668 & 4.7849294 & 4.9622248 \\
10.5 & 0.1647771 & 4.1151315 & 4.2460751 \\
15.0 & 0.0803436 & 2.8801735 & 2.9650878 \\
20.0 & 0.0450998 & 2.1600568 & 2.2215524 \\
25.0 & 0.0288363 & 1.7180541 & 1.7763978 \\
\hline
\end{tabular}


Table 3: Orbit matrices at different energies. Constant coil current. Nominal field.

\begin{tabular}{|c|c|c|c|c|}
\hline \multirow{4}{*}{$\gamma=2.5$} & 0.931162 & 2.908521 & 0.005230 & -0.031102 \\
\hline & -0.045672 & 0.931372 & 0.001339 & -0.023802 \\
\hline & 0.026727 & 0.033443 & 0.937410 & 2.933820 \\
\hline & -0.000214 & -0.004760 & -0.041099 & 0.938256 \\
\hline \multirow[t]{4}{*}{$\gamma=3.6$} & 0.968585 & 2.958826 & 0.002231 & -0.013493 \\
\hline & -0.020964 & 0.968397 & 0.000585 & -0.010310 \\
\hline & 0.009420 & 0.014285 & 0.971257 & 2.969436 \\
\hline & -0.000283 & -0.000436 & -0.019035 & 0.971408 \\
\hline \multirow[t]{4}{*}{$\gamma=4.7$} & 0.982968 & 2.978209 & 0.001102 & -0.007864 \\
\hline & -0.011333 & 0.982994 & 0.000195 & -0.006081 \\
\hline & 0.005958 & 0.008374 & 0.985431 & 2.985071 \\
\hline & 0.000059 & -0.000217 & -0.009698 & 0.985409 \\
\hline \multirow[t]{4}{*}{$\gamma=5.8$} & 0.989175 & 2.986473 & 0.000832 & -0.004820 \\
\hline & -0.007188 & 0.989244 & 0.000275 & -0.003596 \\
\hline & 0.004169 & 0.005456 & 0.990238 & 2.990388 \\
\hline & 0.000088 & -0.000313 & -0.006515 & 0.990186 \\
\hline \multirow[t]{4}{*}{$\gamma=7.0$} & 0.992621 & 2.991047 & 0.000576 & -0.003367 \\
\hline & -0.004910 & 0.992638 & 0.000142 & -0.002591 \\
\hline & 0.002988 & 0.003761 & 0.993073 & 2.993701 \\
\hline & 0.000108 & -0.000224 & -0.004564 & 0.993218 \\
\hline \multirow[t]{4}{*}{$\gamma=8.0$} & 0.994139 & 2.993067 & 0.000599 & -0.002483 \\
\hline & -0.003902 & 0.994148 & 0.000160 & -0.001978 \\
\hline & 0.002356 & 0.002936 & 0.994301 & 2.995083 \\
\hline & 0.000133 & -0.000096 & -0.003721 & 0.994524 \\
\hline \multirow[t]{4}{*}{$\gamma=9.0$} & 0.995407 & 2.994669 & 0.000498 & -0.001916 \\
\hline & -0.003132 & 0.995191 & 0.000147 & -0.001520 \\
\hline & 0.001768 & 0.002125 & 0.995111 & 2.995988 \\
\hline & -0.000035 & -0.000408 & -0.003155 & 0.995413 \\
\hline \multirow[t]{4}{*}{$\gamma=10.5$} & 0.996513 & 2.996168 & 0.000381 & -0.001329 \\
\hline & -0.002398 & 0.996290 & 0.000140 & -0.001031 \\
\hline & 0.001374 & 0.001617 & 0.996236 & 2.997125 \\
\hline & 0.000002 & -0.000295 & -0.002432 & 0.996463 \\
\hline \multirow[t]{4}{*}{$\gamma=15.0$} & 0.998104 & 2.998382 & 0.000139 & -0.000583 \\
\hline & -0.001321 & 0.997930 & 0.000086 & -0.000404 \\
\hline & 0.000787 & 0.000873 & 0.997992 & 2.998914 \\
\hline & 0.000041 & -0.000141 & -0.001301 & 0.998102 \\
\hline \multirow[t]{4}{*}{$\gamma=20.0$} & 0.998807 & 2.999382 & 0.000007 & -0.000319 \\
\hline & -0.000836 & 0.998685 & 0.000037 & -0.000189 \\
\hline & 0.000507 & 0.000529 & 0.998811 & 2.999760 \\
\hline & 0.000042 & -0.000089 & -0.000774 & 0.998866 \\
\hline \multirow[t]{4}{*}{$\gamma=25.0$} & 0.999152 & 2.999878 & -0.000057 & -0.000213 \\
\hline & -0.000595 & 0.999064 & 0.000008 & -0.000106 \\
\hline & 0.000363 & 0.000355 & 0.999214 & 3.000182 \\
\hline & 0.000035 & -0.000071 & -0.000514 & 0.999242 \\
\hline
\end{tabular}




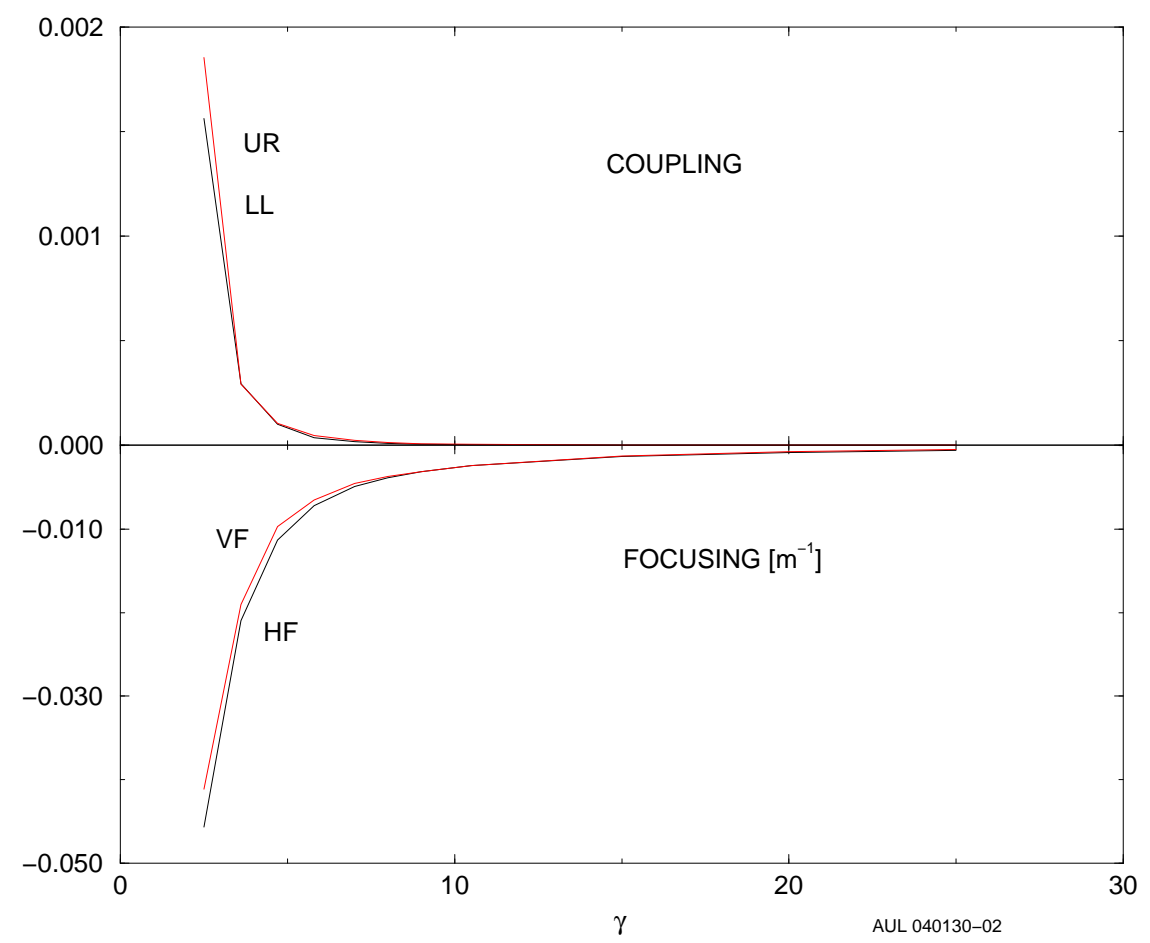

Figure 4: Coupling and Focusing. Constant coil current. Nominal field.

\section{Constant coil current. Enhanced Field}

We repeated the series of cases described in the previous section at constant coil current, but with the current and the field increased by $20 \%{ }^{1}$ The spin maximum rotation at the lowest energy is $\mu=18.834 \mathrm{deg}$, corresponding to a $10.46 \%$ snake, as shown in Fig. 8 . Table 4 shows $\mu$ and the angles of spin axis. The corresponding orbit parameters are shown in Table 5. Focusing and coupling parameters are shown in Fig. 9.

Trajectories at different energies are shown in Fig. 10. Spin component evolution at different energies is shown in Fig. 11. The field on the trajectory for two extreme energies is shown in Fig. 12.

\footnotetext{
${ }^{1}$ Note that since the warm snake is iron dominated, changing the field strength by simple multiplication by a constant may not be realistic, mostly because a real change of current modifies the shape of the fringe field at magnet ends, that finely controls the field integrals. The results in this section and in the next may be less reliable than the results given in the previous section.
}

Table 4: Spin rotation angle and axis angles vs. energy. Constant field increased by $20 \%$.

\begin{tabular}{rrrr}
\hline$\gamma$ & $\mu[\mathrm{deg}]$ & \multicolumn{2}{c}{ axis $\theta, \phi[\mathrm{deg}]$} \\
2.5 & 18.8337113 & 179.9999 & 0.0000467 \\
3.6 & 15.4186568 & 179.9999 & 0.0000414 \\
4.7 & 14.4025087 & 179.9999 & 0.0000404 \\
5.8 & 13.9578618 & 179.9999 & 0.0000396 \\
7.0 & 13.7064577 & 179.9999 & 0.0000390 \\
8.0 & 13.5818027 & 179.9999 & 0.0000385 \\
9.0 & 13.4981131 & 179.9999 & 0.0000383 \\
10.5 & 13.4159296 & 179.9999 & 0.0000381 \\
15.0 & 13.3023335 & 179.9999 & 0.0000379 \\
20.0 & 13.2554927 & 179.9999 & 0.0000378 \\
25.0 & 13.2339939 & 179.9999 & 0.0000378 \\
\hline
\end{tabular}



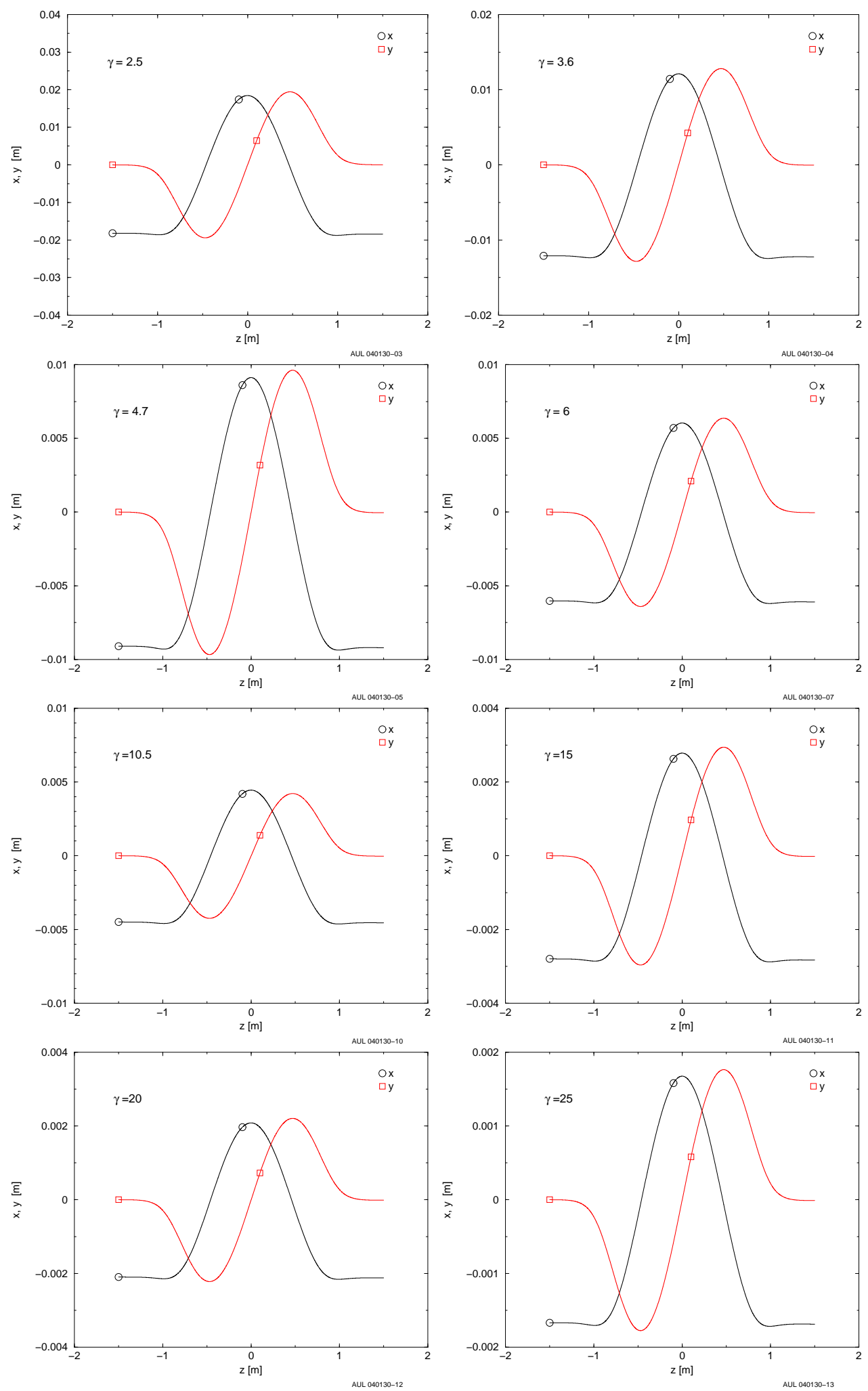

Figure 5: Orbits at constant coil current. Nominal field - Note the changes of vertical scale. 

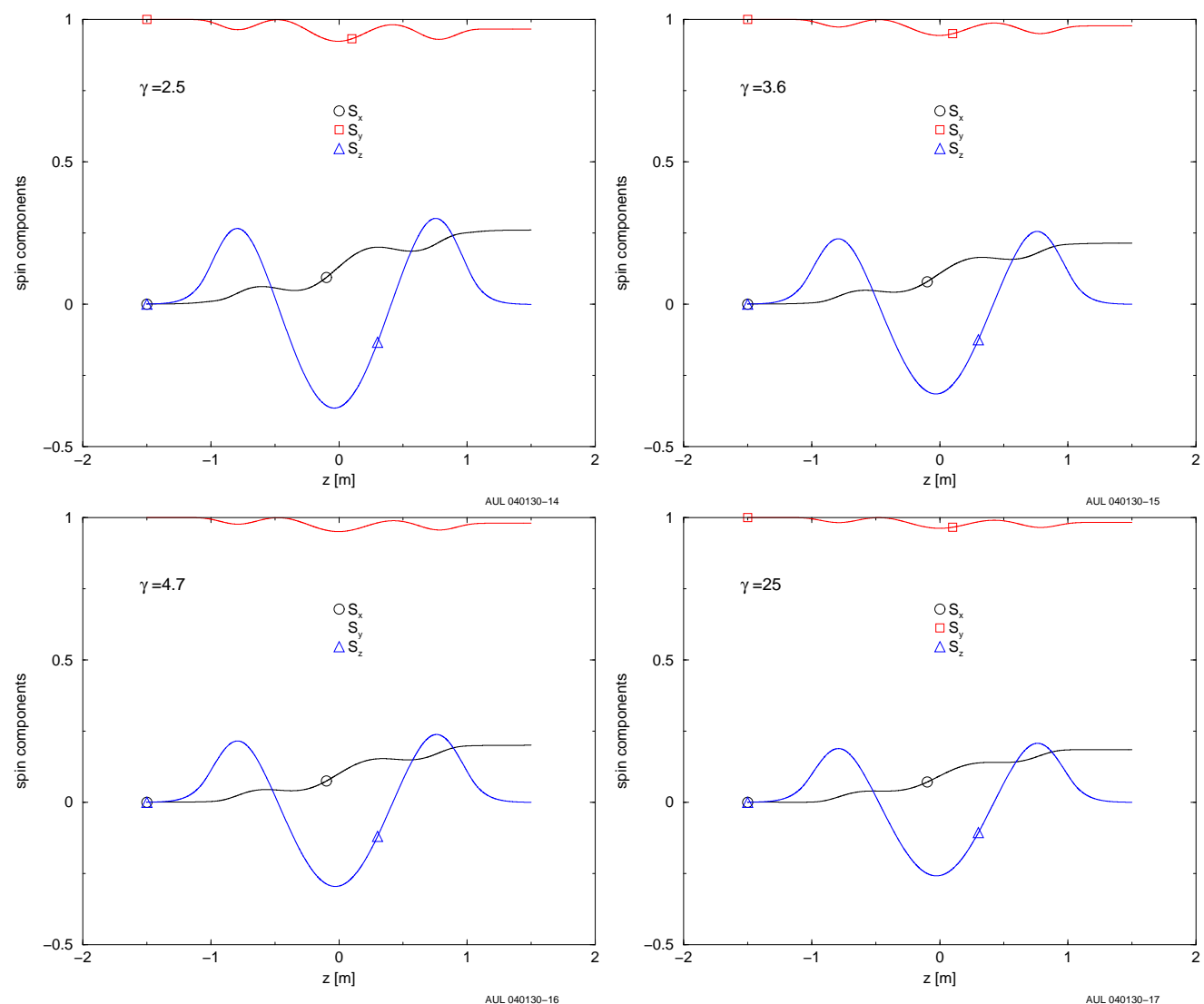

Figure 6: Spin components. Constant coil current. Nominal field - Spin is injected vertical.
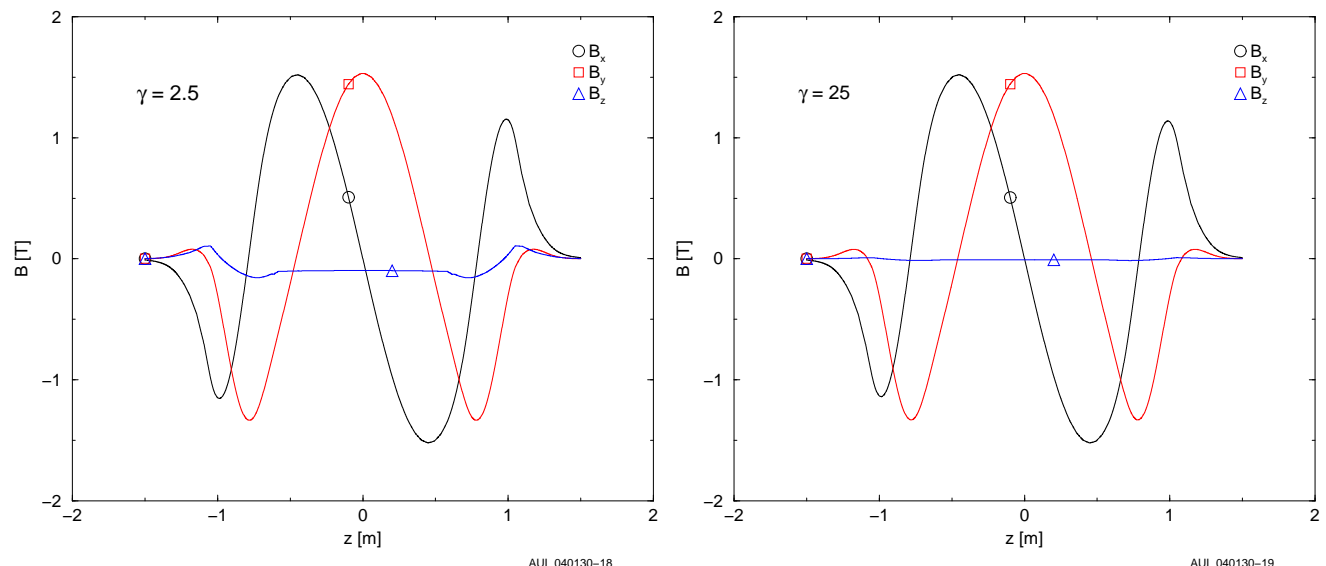

Figure 7: Field components. Constant current - The longitudinal field mostly shows at low energy. 


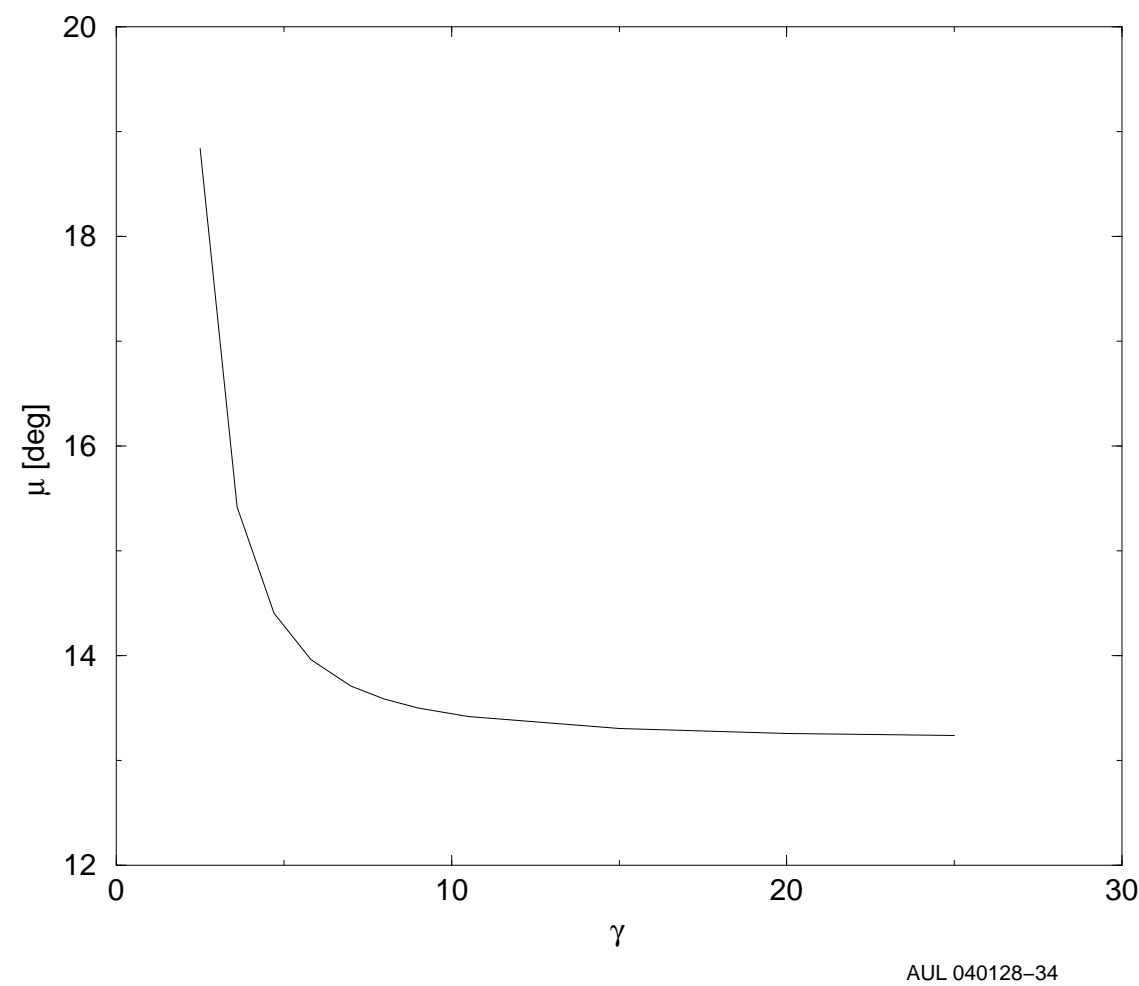

Figure 8: Spin rotation angle $\mu$ vs. beam energy. Constant field increased by $20 \%$.

Table 5: Orbit lengthening and max excursion vs, energy. Constant field increased by $20 \%$.

\begin{tabular}{rrrr}
\hline$\gamma$ & $d s[\mathrm{~mm}]$ & \multicolumn{2}{c}{$\max x$ and $y[\mathrm{~mm}]$} \\
2.5 & 4.3488532 & 21.1466355 & 21.8262279 \\
3.6 & 1.8948347 & 13.9056654 & 14.3796388 \\
4.7 & 1.0717843 & 10.4993089 & 10.8223750 \\
5.8 & 0.6917238 & 8.3895478 & 8.6967083 \\
7.0 & 0.4700528 & 6.9463046 & 7.1701444 \\
8.0 & 0.3580148 & 6.0210906 & 6.2579575 \\
9.0 & 0.2818698 & 5.3712775 & 5.5530157 \\
10.5 & 0.2063542 & 4.6289754 & 4.7515491 \\
15.0 & 0.1006099 & 3.2097776 & 3.3180022 \\
20.0 & 0.0564745 & 2.4072075 & 2.4859517 \\
25.0 & 0.0361086 & 1.9237555 & 1.9878106 \\
\hline
\end{tabular}




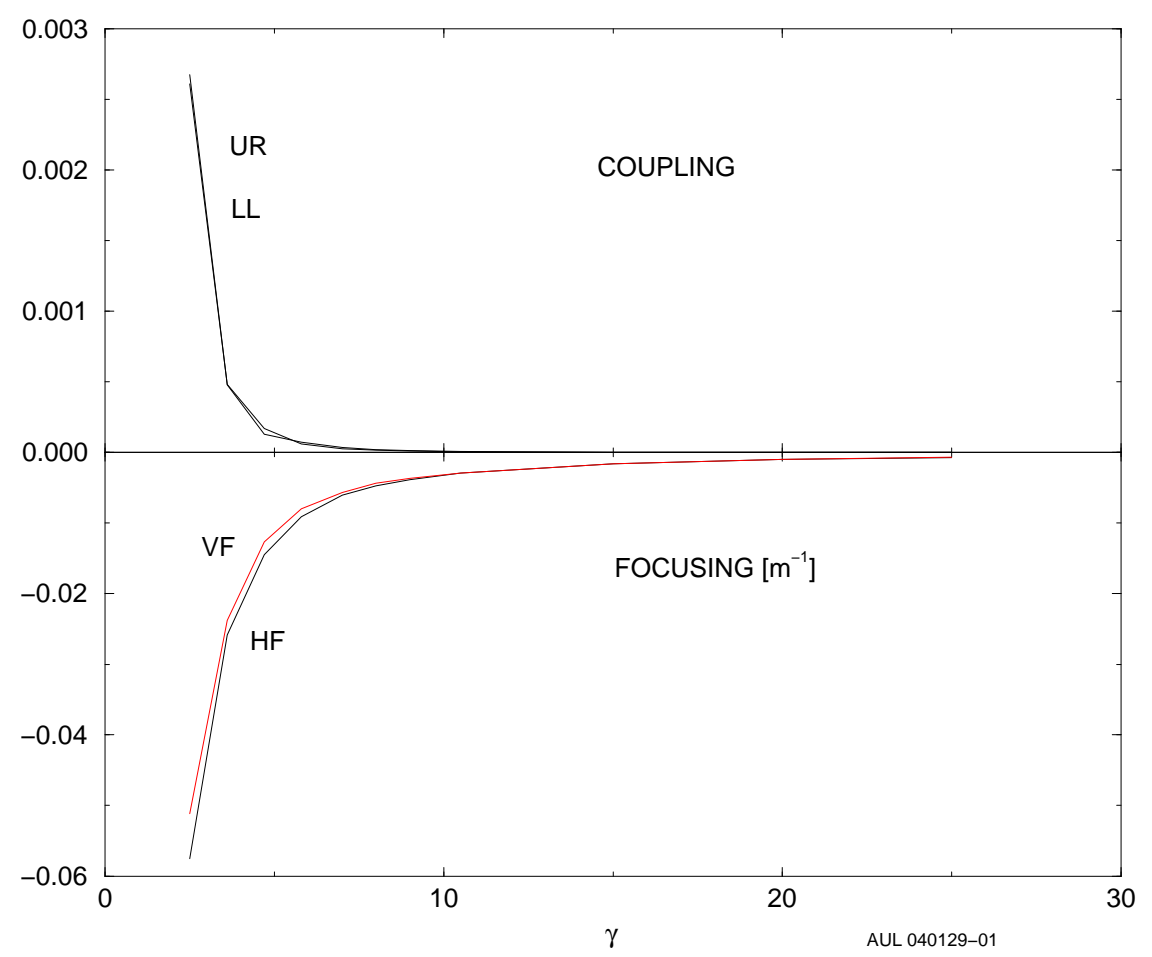

Figure 9: Coupling and focusing. Constant field increased by $20 \%$.
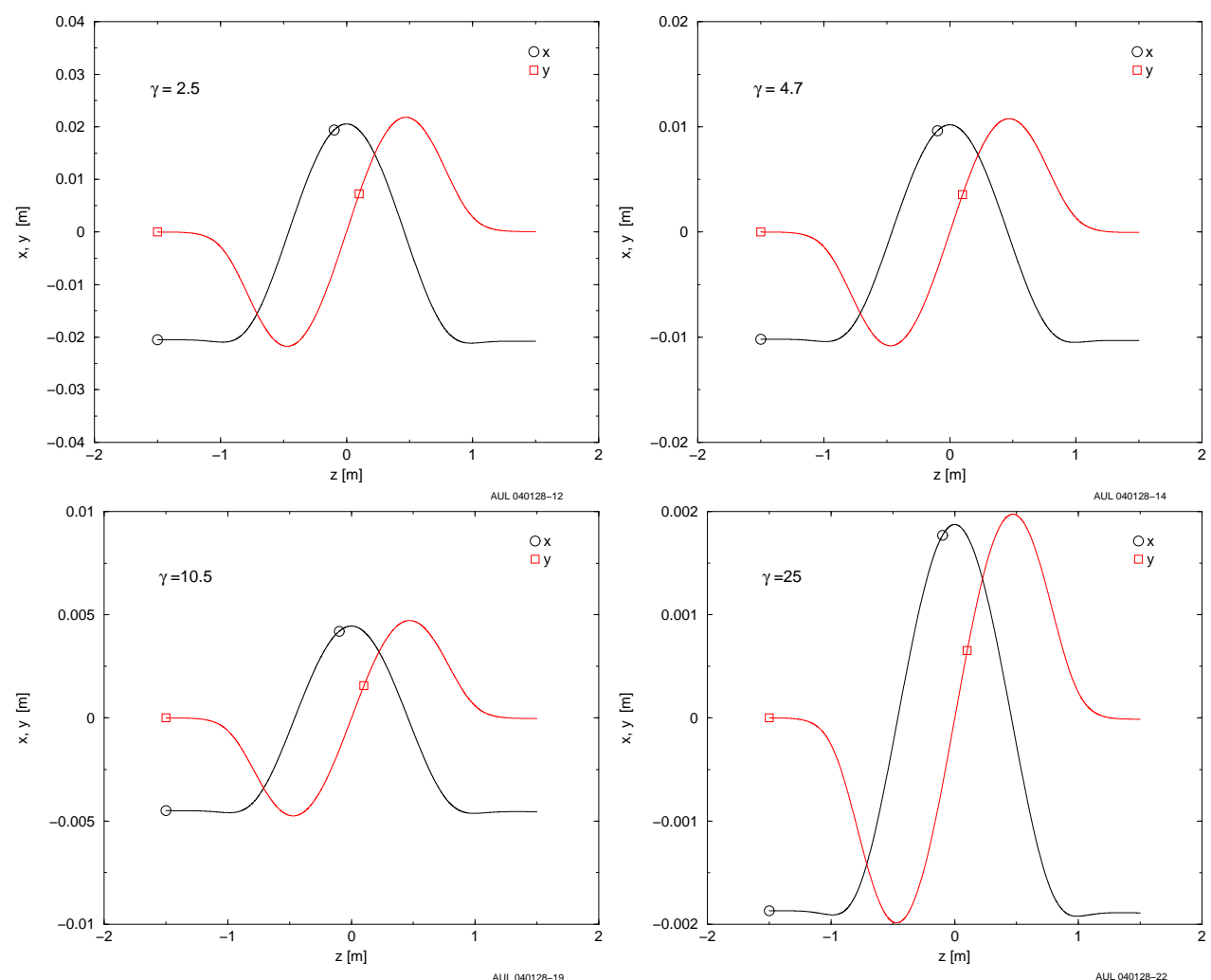

Figure 10: Orbits at field increased by $20 \%$. 

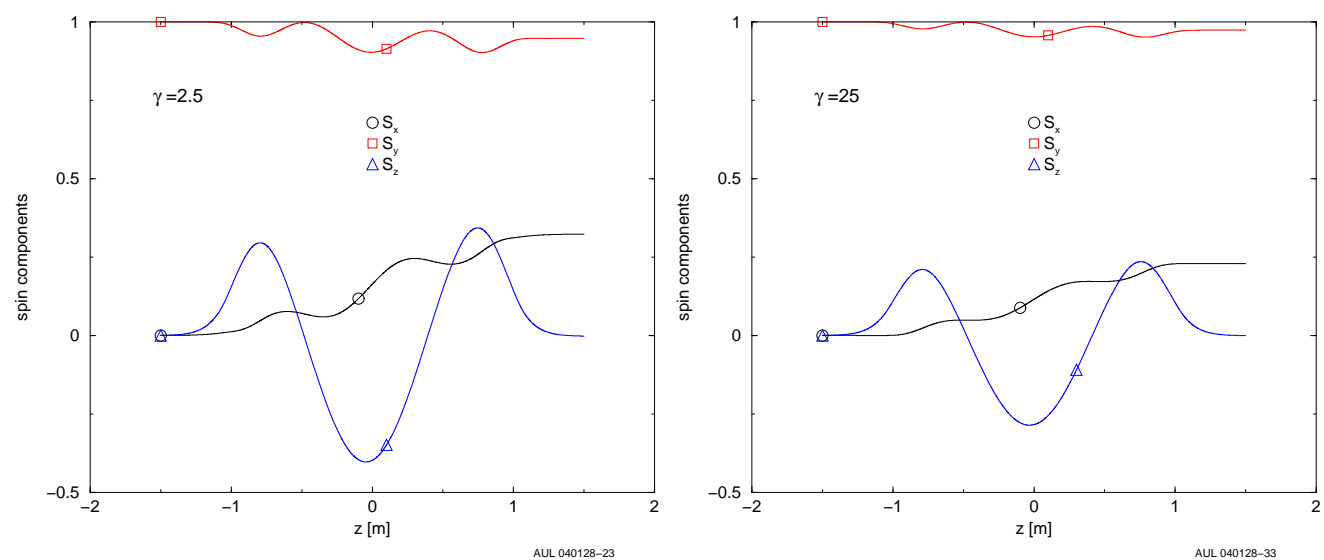

Figure 11: Spin components at field increased by $20 \%$.
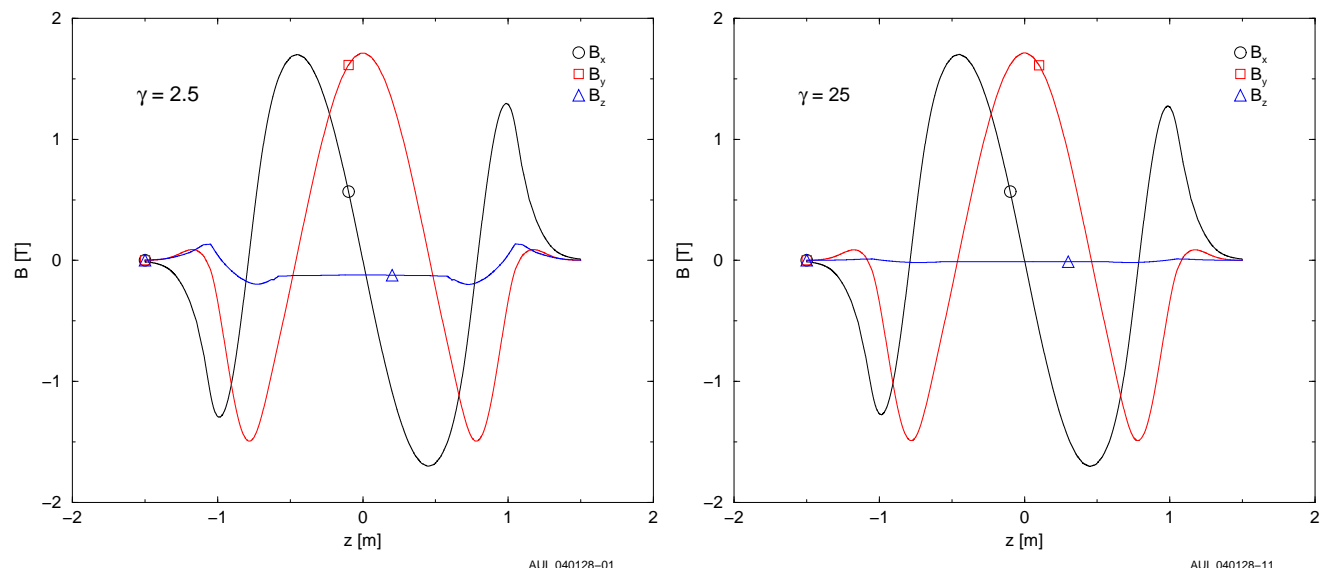

Figure 12: Field components at field increased by $20 \%$.

Orbit matrices for different energies are given in Table 6 .

\section{Constant spin rotation angle $\mu$}

A further series was done keeping constant the rotation angle of spin at an "average" value $\mu \approx$ $12 \mathrm{deg}$, corresponding to a $6.7 \%$ snake. This was obtained by changing the nominal value of the field $B_{0}=1.5 T$ as a function of energy. Results are shown in Table 7: spin rotation angle and axis angles. The corresponding orbit parameters are shown in Table 8.

The field along the trajectory and the trajectory of a central particle at the energy $\gamma=2.5$ (injection in the AGS) are shown in Fig. 13. The longitudinal field responsible for part of the coupling, shows up at this energy. This $B_{z}$ is however much smaller than the corresponding component in the cold snake [4].

Trajectories at various proton energies are shown in Fig. 14. $x$ and $y$ are in meters. Focusing and coupling are shown in Fig. 15. Orbit matrices for different energies are given in Table 9.

\section{Conclusions}

The warm snake, as represened by numerical field map, is doing what is supposed to do, i.e. it rotates the spin by an angle larger that the present solenoidal snake in the AGS. Focusing in both planes is a problem, however the warm snake has much less focusing that the cold snake. Coupling 
Table 6: Orbit matrices at different energies at field increased by $20 \%$.

\begin{tabular}{|c|c|c|c|c|}
\hline \multirow[t]{4}{*}{$\gamma=2.5$} & 0.913362 & 2.883916 & 0.005954 & -0.040187 \\
\hline & -0.057537 & 0.913322 & 0.001125 & -0.030976 \\
\hline & 0.031071 & 0.041057 & 0.922395 & 2.916451 \\
\hline & -0.000709 & -0.004800 & -0.051167 & 0.922496 \\
\hline \multirow[t]{4}{*}{$\gamma=3.6$} & 0.961224 & 2.948738 & 0.003259 & -0.017043 \\
\hline & -0.025853 & 0.961051 & 0.000715 & -0.013449 \\
\hline & 0.012758 & 0.017710 & 0.964425 & 2.961885 \\
\hline & -0.000426 & -0.001697 & -0.023752 & 0.963970 \\
\hline \multirow[t]{4}{*}{$\gamma=4.7$} & 0.978351 & 2.972137 & 0.001140 & -0.010606 \\
\hline & -0.014477 & 0.978144 & 0.000144 & -0.007418 \\
\hline & 0.005362 & 0.009974 & 0.980793 & 2.972522 \\
\hline & -0.000306 & 0.000684 & -0.012665 & 0.977851 \\
\hline \multirow[t]{4}{*}{$\gamma=5.8$} & 0.986293 & 2.982619 & 0.001073 & -0.006091 \\
\hline & -0.009120 & 0.986320 & 0.000318 & -0.004616 \\
\hline & 0.005055 & 0.006875 & 0.988088 & 2.987951 \\
\hline & 0.000122 & -0.000190 & -0.007959 & 0.987989 \\
\hline \multirow[t]{4}{*}{$\gamma=7.0$} & 0.990960 & 2.988814 & 0.000626 & -0.004183 \\
\hline & -0.006005 & 0.991013 & 0.000190 & -0.003111 \\
\hline & 0.003687 & 0.004671 & 0.991509 & 2.991864 \\
\hline & 0.000096 & -0.000340 & -0.005642 & 0.991542 \\
\hline \multirow[t]{4}{*}{$\gamma=8.0$} & 0.992948 & 2.991466 & 0.000599 & -0.003189 \\
\hline & -0.004694 & 0.992960 & 0.000150 & -0.002477 \\
\hline & 0.002874 & 0.003599 & 0.993401 & 2.994060 \\
\hline & 0.000112 & -0.000210 & -0.004343 & 0.993554 \\
\hline \multirow[t]{4}{*}{$\gamma=9.0$} & 0.994208 & 2.993131 & 0.000599 & -0.002451 \\
\hline & -0.003857 & 0.994216 & 0.000156 & -0.001964 \\
\hline & 0.002321 & 0.002897 & 0.994359 & 2.995128 \\
\hline & 0.000133 & -0.000088 & -0.003683 & 0.994581 \\
\hline \multirow[t]{4}{*}{$\gamma=10.5$} & 0.995747 & 2.994800 & 0.000474 & -0.001732 \\
\hline & -0.002906 & 0.995535 & 0.000146 & -0.001377 \\
\hline & 0.001645 & 0.001967 & 0.995451 & 2.996319 \\
\hline & -0.000022 & -0.000862 & -0.002935 & 0.995734 \\
\hline \multirow[t]{4}{*}{$\gamma=15$} & 0.997714 & 2.997833 & 0.000203 & -0.000750 \\
\hline & -0.001588 & 0.997519 & 0.000105 & -0.000542 \\
\hline & 0.000936 & 0.001059 & 0.997548 & 2.998457 \\
\hline & 0.000035 & -0.000177 & -0.001588 & 0.997686 \\
\hline \multirow[t]{4}{*}{$\gamma=20.0$} & 0.998575 & 2.999050 & 0.000049 & -0.000401 \\
\hline & -0.000997 & 0.998433 & 0.000054 & -0.000253 \\
\hline & 0.000603 & 0.000644 & 0.998540 & 2.999478 \\
\hline & 0.000043 & -0.000106 & -0.000949 & 0.998612 \\
\hline \multirow[t]{4}{*}{$\gamma=25.0$} & 0.998994 & 2.999652 & -0.000029 & -0.000260 \\
\hline & -0.000705 & 0.998890 & 0.000021 & -0.000141 \\
\hline & 0.000430 & 0.000434 & 0.999032 & 2.999990 \\
\hline & 0.000039 & -0.000079 & -0.000632 & 0.999071 \\
\hline
\end{tabular}


Table 7: Spin rotation angle and axis angles vs. energy. Constant $\mu$.

\begin{tabular}{rrrrr}
\hline$\gamma$ & $B / B_{0}$ & $\mu[\mathrm{deg}]$ & \multicolumn{2}{c}{ axis $\theta, \phi[\mathrm{deg}]$} \\
2.5 & 0.900 & 12.2110 & 179.9999 & 0.0000553 \\
3.6 & 0.990 & 12.1028 & 179.9999 & 0.0000477 \\
4.7 & 1.020 & 11.9979 & 179.9999 & 0.0000450 \\
5.8 & 1.035 & 11.9694 & 179.9999 & 0.0000436 \\
7.0 & 1.047 & 12.0249 & 179.9999 & 0.0000422 \\
8.0 & 1.050 & 11.9833 & 179.9999 & 0.0000418 \\
9.0 & 1.060 & 12.1341 & 179.9999 & 0.0000410 \\
10.5 & 1.061 & 12.0829 & 179.9999 & 0.0000408 \\
15.0 & 1.061 & 11.9809 & 179.9999 & 0.0000406 \\
20.0 & 1.062 & 11.9610 & 179.9999 & 0.0000405 \\
25.0 & 1.065 & 12.0081 & 179.9999 & 0.0000402 \\
\hline
\end{tabular}

Table 8: Orbit lengthening and max excursion vs. energy. Constant $\mu$.

\begin{tabular}{rrrrr}
$\gamma$ & $B / B_{0}$ & $d s[\mathrm{~mm}]$ & \multicolumn{2}{c}{$\max x$ and $y[\mathrm{~mm}]$} \\
2.5 & 0.9 & 2.8003585 & 16.9573089 & 17.4816786 \\
3.6 & 0.99 & 1.4812035 & 12.3536674 & 12.7181048 \\
4.7 & 1.02 & 0.8900438 & 9.4732771 & 9.8634862 \\
5.8 & 1.035 & 0.5915857 & 7.8205709 & 8.0432523 \\
7.0 & 1.047 & 0.4114369 & 6.4835136 & 6.7084318 \\
8.0 & 1.05 & 0.3151799 & 5.7598456 & 5.8719177 \\
9.0 & 1.06 & 0.2529059 & 5.1429520 & 5.2601193 \\
10.5 & 1.061 & 0.1855046 & 4.4221150 & 4.5051929 \\
15.0 & 1.061 & 0.0904474 & 3.0850448 & 3.1459986 \\
20.0 & 1.062 & 0.0508665 & 2.3637192 & 2.3593166 \\
25.0 & 1.065 & 0.0327072 & 1.8311676 & 1.8918751 \\
\hline
\end{tabular}

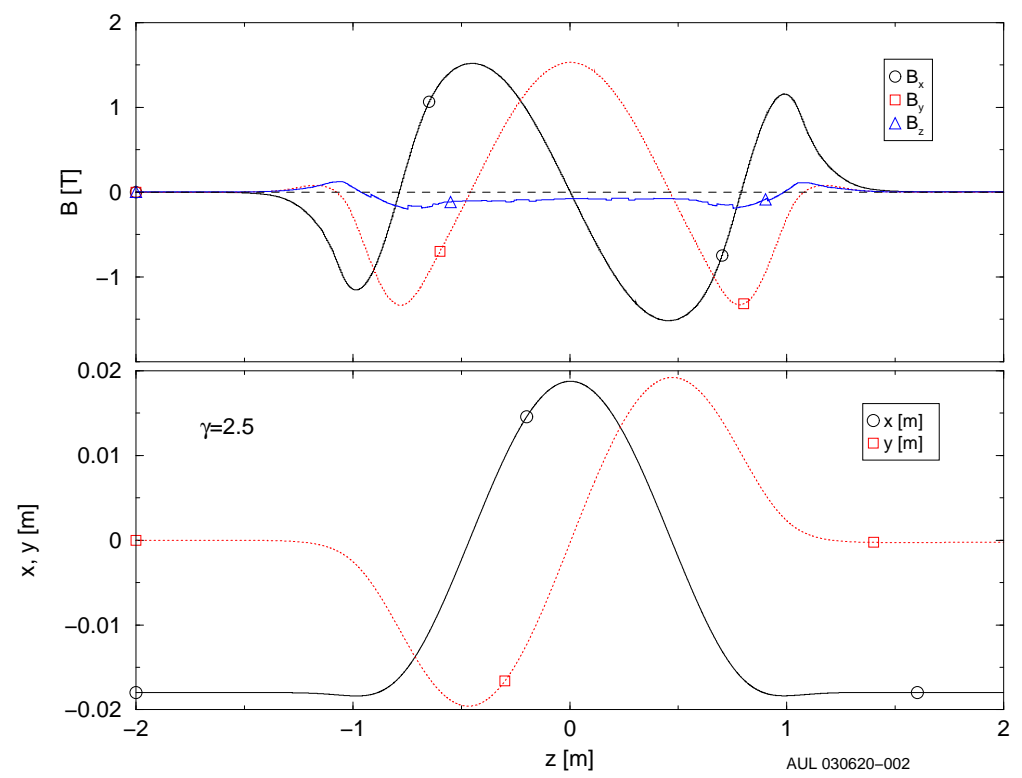

Figure 13: field on trajectory and trajectory for $\gamma=2.5$. Constant $\mu$. 

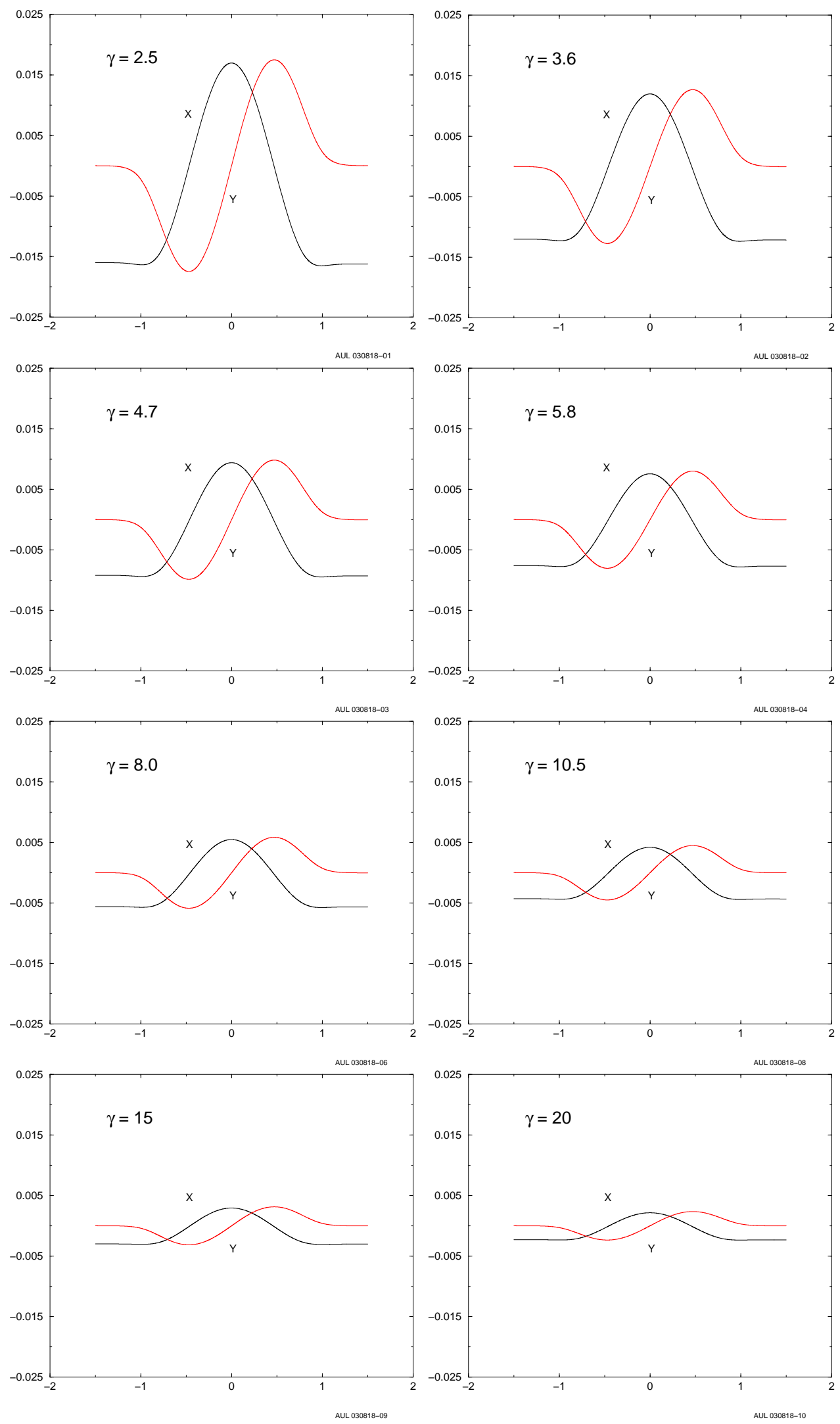

Figure 14: Trajectory for different energies. Constant $\mu$. 


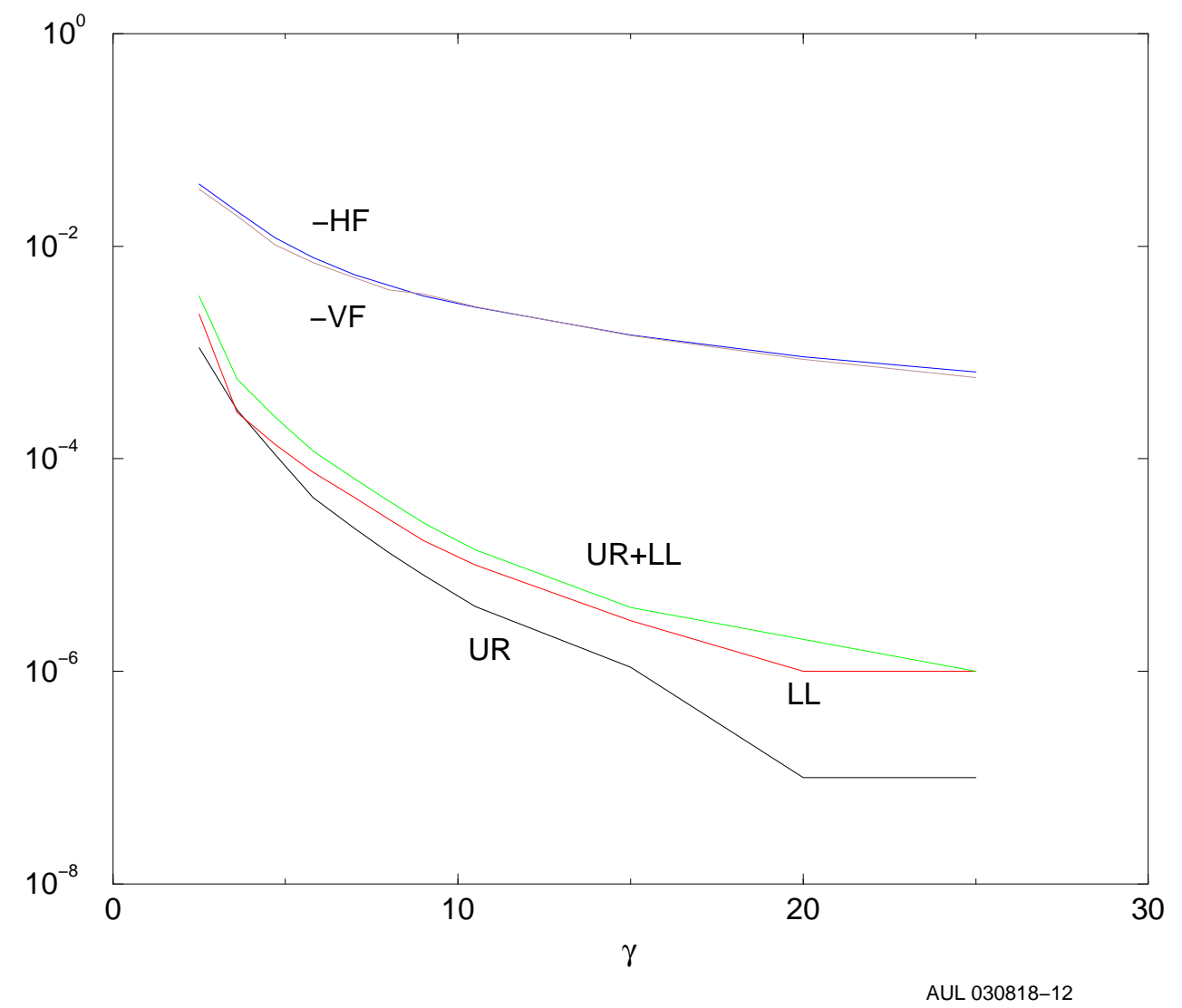

Figure 15: Focusing and coupling parameters for different energies. Constant $\mu$. 
Table 9: Orbit matrices at different energies. Constant $\mu$.

\begin{tabular}{|c|c|c|c|c|}
\hline \multirow[t]{4}{*}{$\gamma=2.5$} & 0.945140 & 2.927443 & 0.003425 & -0.025866 \\
\hline & -0.036400 & 0.945382 & 0.000662 & -0.019693 \\
\hline & 0.022669 & 0.026702 & 0.950662 & 2.948036 \\
\hline & -0.000296 & -0.005292 & -0.032619 & 0.950831 \\
\hline \multirow[t]{4}{*}{$\gamma=3.6$} & 0.969200 & 2.959670 & 0.002181 & -0.013225 \\
\hline & -0.020557 & 0.969034 & 0.000567 & -0.010118 \\
\hline & 0.009189 & 0.014120 & 0.971896 & 2.970149 \\
\hline & -0.000261 & -0.000102 & -0.018603 & 0.972076 \\
\hline \multirow[t]{4}{*}{$\gamma=4.7$} & 0.982279 & 2.977312 & 0.001092 & -0.008214 \\
\hline & -0.011789 & 0.982312 & 0.000180 & -0.006339 \\
\hline & 0.006182 & 0.008705 & 0.984838 & 2.984422 \\
\hline & 0.000053 & -0.000233 & -0.010084 & 0.984840 \\
\hline \multirow[t]{4}{*}{$\gamma=5.8$} & 0.988334 & 2.985323 & 0.000927 & -0.005157 \\
\hline & -0.007755 & 0.988381 & 0.000300 & -0.003873 \\
\hline & 0.004422 & 0.005892 & 0.989648 & 2.989698 \\
\hline & 0.000117 & -0.000223 & -0.006917 & 0.989566 \\
\hline \multirow[t]{4}{*}{$\gamma=7.0$} & 0.991991 & 2.990214 & 0.000554 & -0.003698 \\
\hline & -0.005325 & 0.992024 & 0.000149 & -0.002782 \\
\hline & 0.003249 & 0.004109 & 0.992418 & 2.992927 \\
\hline & 0.000104 & -0.000261 & -0.005011 & 0.992528 \\
\hline \multirow[t]{4}{*}{$\gamma=8.0$} & 0.993655 & 2.992387 & 0.000619 & -0.002788 \\
\hline & -0.004229 & 0.993651 & 0.000145 & -0.002232 \\
\hline & 0.002557 & 0.003192 & 0.994101 & 2.994856 \\
\hline & 0.000116 & -0.000158 & -0.003870 & 0.994276 \\
\hline \multirow[t]{4}{*}{$\gamma=9.0$} & 0.994941 & 2.994098 & 0.000559 & -0.002188 \\
\hline & -0.003386 & 0.994894 & 0.000148 & -0.001760 \\
\hline & 0.002038 & 0.002607 & 0.994550 & 2.995346 \\
\hline & 0.000122 & -0.000027 & -0.003540 & 0.994819 \\
\hline \multirow[t]{4}{*}{$\gamma=10.5$} & 0.996130 & 2.995644 & 0.000440 & -0.001528 \\
\hline & -0.002651 & 0.995912 & 0.000145 & -0.001211 \\
\hline & 0.001509 & 0.001796 & 0.995839 & 2.996733 \\
\hline & -0.000008 & -0.000326 & -0.002685 & 0.996099 \\
\hline \multirow[t]{4}{*}{$\gamma=15$} & 0.997911 & 2.998107 & 0.000180 & -0.000662 \\
\hline & -0.001453 & 0.997727 & 0.000097 & -0.000476 \\
\hline & 0.000860 & 0.000967 & 0.997766 & 2.998688 \\
\hline & 0.000039 & -0.000155 & -0.001446 & 0.997894 \\
\hline \multirow[t]{4}{*}{$\gamma=20.0$} & 0.998694 & 2.999217 & 0.000045 & -0.000353 \\
\hline & -0.000914 & 0.998563 & 0.000050 & -0.000225 \\
\hline & 0.000551 & 0.000589 & 0.998668 & 2.999621 \\
\hline & 0.000043 & -0.000090 & -0.000865 & 0.998737 \\
\hline \multirow[t]{4}{*}{$\gamma=25.0$} & 0.999067 & 2.999756 & -0.000042 & -0.000238 \\
\hline & -0.000654 & 0.998971 & 0.000015 & -0.000124 \\
\hline & 0.000399 & 0.000398 & 0.999116 & 3.000079 \\
\hline & 0.000037 & -0.000075 & -0.000577 & 0.999151 \\
\hline
\end{tabular}


is also relatively small, because in this design the longitudinal component of the magnetic field is small. We do not plan a correction solenoid as in the cold snake.

The snake was built and delivered to BNL. It has successfully passed a series of electrical tests and field measurements in our Magnet Test facility, and is due for installation on the AGS by the end of February 2004. We will promptly compare the measured field with the ideal one shown in this report. A preliminary comparison seems in good agreement.

Insertion of the warm snake in the AGS lattice doesn't seem to create problems that cannot be controlled. Since the horizontal orbit starts off axis, the AGS will require horizontal steering. Numerical work is in progress and results will be shown on a further report.

\section{References}

[1] M.Okamura, T.Katayama, T.Tominaka T.Ohkawa R.Gupta A.U.Luccio W.W.MacKay T.Roser E.WILlen: Design of a partial snake for the AGS. In: European Particle Accelerator Conference, EPAC2002, 2421, Paris, France, 3-7 June 2002.

[2] T.Roser, M.Syphers, E.Courant L.Ratner M.Okamura: Helical Partial Snake for the $A G S$. Technical Report AGS/RHIC/SN 072, Brookhaven National Laboratory. Upton, NY, March 191998.

[3] R.Gupta, A.Luccio, G.Morgan W.Mackay K.Power T.Roser E.Willen M.Okamura: Magnetic design of a super conducting AGS snake. In: Particle Accelerator Conference, paper:WPAE002, Portland, Oregon, 2003.

[4] A.U.Luccio: Cold AGS Snake Optimization by Modeling. Technical Report C-A/AP/128, Brookhaven National Laboratory. Upton, NY, Upton, NY, December 2003.

[5] A.U.Luccio: Numerical Optimization of Siberian Snakes and Spin Rotators for RHIC. In: Trends in Collider Spin Physics, 244, Trieste, Italy, 5-8 Dec 1995. World Scientific.

[6] Vector Fields, Ltd: Opera-3d Reference Manual. Technical report, England, January 2002.

[7] H.Grote and F.Ch.Iselin: The MAD program, Vers.8.19. Technical Report CERN/SL/90-13, European Organization for Nuclear Research, Geneva, CH, 1996.

[8] A.U.Luccio: Angles from Spin Matrices. Technical Report AGS/RHIC/SN No. 03, Brookhaven National Laboratory. Upton, NY, October 81996. 